\title{
New Designs of Reduced-Order Observer-Based Controllers for Singularly Perturbed Linear Systems
}

\author{
Heonjong Yoo and Zoran Gajic \\ Department of Electrical and Computer Engineering, Rutgers University, 94 Brett Road, Piscataway, NJ, USA \\ Correspondence should be addressed to Zoran Gajic; gajic@ece.rutgers.edu
}

Received 28 May 2017; Revised 18 September 2017; Accepted 18 October 2017; Published 13 December 2017

Academic Editor: Yan-Wu Wang

Copyright (c) 2017 Heonjong Yoo and Zoran Gajic. This is an open access article distributed under the Creative Commons Attribution License, which permits unrestricted use, distribution, and reproduction in any medium, provided the original work is properly cited.

\begin{abstract}
The slow and fast reduced-order observers and reduced-order observer-based controllers are designed by using the two-stage feedback design technique for slow and fast subsystems. The new designs produce an arbitrary order of accuracy, while the previously known designs produce the accuracy of $O(\epsilon)$ only where $\epsilon$ is a small singular perturbation parameter. Several cases of reduced-order observer designs are considered depending on the measured state space variables: only all slow variables are measured, only all fast variables are measured, and some combinations of the slow and fast variables are measured. Since the two-stage methods have been used to overcome the numerical ill-conditioning problem for Cases (III)-(V), they have similar procedures. The numerical ill-conditioning problem is avoided so that independent feedback controllers can be applied to each subsystem. The design allows complete time-scale separation for both the reduced-order observer and controller through the complete and exact decomposition into slow and fast time scales. This method reduces both offline and online computations.
\end{abstract}

\section{Introduction}

The presentation in this paper is based on the doctoral dissertation [1]. The fundamental technique used is the two-stage feedback controller design $[2,3]$. The full-order observers for singularly perturbed linear systems were considered in [4-10]. The reduced-order observer for singularly perturbed discrete-time systems has been studied only in two papers [11, 12], both of them producing accuracy of $O(\epsilon)$ (an $O(\epsilon)$ is defined by $O(\epsilon) \leq k \epsilon$, where $k$ is a bounded constant), where $\epsilon$ is a small singular perturbation parameter. There are no corresponding results reported in the literature for the reduced-order continuous time observers. Since $O(\epsilon)$ accuracy might not be acceptable, our motivation is to design reduced-order observers for this class of systems with the accuracy of $O\left(\epsilon^{i}\right) \leq c \epsilon^{i}$, $i=1,2,3, \ldots$, where $\epsilon$ is a bounded constant, which is along the lines of high accuracy techniques for singularly perturbed systems [5]. Note that, for $\epsilon<1, \epsilon^{i} \rightarrow 0$ rapidly.
Consider a singularly perturbed linear system that contains slow and fast modes [6]:

$$
\begin{aligned}
E \dot{x}(t) & =A x(t) \\
y(t) & =C x(t) \\
{\left[\begin{array}{cc}
I_{n_{1}} & 0 \\
0 & \epsilon I_{n_{2}}
\end{array}\right]\left[\begin{array}{c}
\dot{x}_{1}(t) \\
\dot{x}_{2}(t)
\end{array}\right] } & =\left[\begin{array}{ll}
A_{11} & A_{12} \\
A_{21} & A_{22}
\end{array}\right]\left[\begin{array}{l}
x_{1}(t) \\
x_{2}(t)
\end{array}\right], \\
y(t) & =\left[\begin{array}{ll}
C_{1} & C_{2}
\end{array}\right]\left[\begin{array}{l}
x_{1}(t) \\
x_{2}(t)
\end{array}\right],
\end{aligned}
$$

where $\epsilon$ is a small positive singular perturbation parameter that indicates separation of state variables $x(t) \in \mathfrak{R}^{n}$ into slow $x_{1}(t) \in \mathfrak{R}^{n_{1}}$ and fast $x_{2}(t) \in \mathfrak{R}^{n_{2}}$ and $n_{1}+n_{2}=n$, and $y(t) \in$ $\mathfrak{R}^{l}$ are the system measurements. The problem matrices are constant and of appropriate dimensions. It was assumed that the matrix $C$ has full rank equal to $l$. The singularly perturbed system is studied under the following standard assumption [4]. 
Assumption 1. $A_{22}$ is nonsingular.

In the following we consider five cases for the reducedorder observer-based controller design for singularly perturbed linear systems depending on combinations of measured states [1].

\section{Case I: Controller Design When Only All Slow Variables Are Measured}

Consider the linear time invariant singularly perturbed control system [1], in which only slow variables are directly measured:

$$
\begin{aligned}
& \dot{x}_{1}(t)=A_{11} x_{1}(t)+A_{12} x_{2}(t)+B_{1} u(t) \\
& \dot{x}_{2}(t)=\frac{1}{\epsilon} A_{21} x_{1}(t)+\frac{1}{\epsilon} A_{22} x_{2}(t)+\frac{1}{\epsilon} B_{2} u(t) \\
& y(t)=x_{1}(t),
\end{aligned}
$$

where $u(t) \in \Re^{m}$ is the control input. The reduced-order observer for (2) is given by [13]

$$
\epsilon \dot{\bar{z}}_{2}(t)=A_{z} \widehat{z}_{2}(t)+B_{z} u(t)+K_{z} y(t)
$$

where

$$
\begin{aligned}
& A_{z}=A_{22}-K_{11} A_{12}, \\
& B_{z}=B_{2}-K_{11} B_{1} \\
& K_{z}=A_{21}-K_{11} A_{11}+\frac{1}{\epsilon} A_{22} K_{11}-\frac{1}{\epsilon} K_{11} A_{12} K_{11} .
\end{aligned}
$$

The state estimation of the fast variables is obtained from

$$
\widehat{x}_{2}(t)=\widehat{z}_{2}(t)+\frac{1}{\epsilon} K_{11} y(t)
$$

so that

$$
\widehat{x}(t)=\left[\begin{array}{l}
x_{1}(t) \\
\widehat{x}_{2}(t)
\end{array}\right]=\left[\begin{array}{c}
y(t) \\
\widehat{x}_{2}(t)
\end{array}\right] .
$$

The matrix $K_{11}$ is chosen to stabilize the reduced-order observer (3); that is,

$$
\lambda\left(A_{22}-K_{11} A_{12}\right)=\lambda\left(A_{22}^{T}-A_{12}^{T} K_{11}^{T}\right)=\lambda_{\text {robs }}^{\text {desired }} .
$$

To design this reduced-order observer the following assumption is needed [1].

Assumption 2. The pair $\left(A_{22}^{T}, A_{12}^{T}\right)$ is controllable, which is equivalent to the pair $\left(A_{22}, A_{12}\right)$ being observable, which is equivalent to the requirement that the original system is observable.

In the following, the Chang transformation matrix [4] will be needed:

$$
\begin{aligned}
T_{c} & =\left[\begin{array}{cc}
I_{n} & \epsilon H \\
-L & I_{m}-\epsilon L H
\end{array}\right], \\
T_{c}^{-1} & =\left[\begin{array}{cc}
I_{n}-\epsilon H L & -\epsilon H \\
L & I_{m}
\end{array}\right],
\end{aligned}
$$

where matrices $L$ and $H$ satisfy the algebraic equations

$$
\begin{aligned}
& 0=\epsilon L\left(A_{11}-A_{12} L\right)+\left(A_{21}-A_{22} L\right) \\
& 0=\epsilon\left(A_{11}-A_{12} L\right) H+A_{12}-H\left(A_{22}+\epsilon L A_{12}\right) .
\end{aligned}
$$

The solutions for $L$ and $H$ can be obtained using either the fixed-point iterations or Newton method or eigenvector method [5].

Using the separation principle, the observer-based controller design via the two-stage design [2] produces [1]

$$
\begin{aligned}
u(t) & =-F \widehat{x}(t)=-\left[\begin{array}{ll}
F_{1} & F_{2}
\end{array}\right]\left[\begin{array}{l}
x_{1}(t) \\
\widehat{x}_{2}(t)
\end{array}\right] \\
& =-\left[\begin{array}{ll}
F_{s}+F_{f 2} P & F_{f 2}
\end{array}\right] T_{c}^{-1}\left[\begin{array}{c}
y(t) \\
\widehat{x}_{2}(t)
\end{array}\right] .
\end{aligned}
$$

The feedback gain $F_{s}$ is chosen thus to place slow eigenvalues at the desired locations; that is,

$$
\lambda\left(A_{s}-B_{s} F_{s}\right)=\lambda_{s}^{\text {desired }} .
$$

The matrix $P$ is obtained from the Sylvester algebraic equation

$$
\begin{aligned}
\epsilon P\left(A_{s}-B_{s} F_{s}\right)-B_{f} F_{s}-A_{f} P & =0 \Longrightarrow \\
P^{(0)} & =A_{f}^{-1} B_{f} F_{s},
\end{aligned}
$$

where

$$
\begin{aligned}
& A_{s}=A_{11}-A_{12} L, \\
& B_{s}=B_{1}\left(I_{n}-\epsilon H L\right)-\epsilon H B_{2} \\
& A_{f}=A_{22}+\epsilon L A_{12}, \\
& B_{f}=\epsilon L B_{1}+B_{2} .
\end{aligned}
$$

The feedback gain $F_{f 2}$ is chosen thus to place the fast eigenvalues at the desired locations; that is,

$$
\begin{gathered}
\lambda\left(A_{f}-\left(B_{f}+\epsilon P B_{s}\right) F_{f 2}\right)=\lambda\left(A_{f}-B_{f \text { new }} F_{f 2}\right) \\
=\lambda_{f}^{\text {desired }} .
\end{gathered}
$$

To obtain the reduced-order feedback gains $F_{s}$ and $F_{f 2}$, the following controllability assumption is needed [14].

Assumption 3. The pairs $\left(A_{s}, B_{s}\right)$ and $\left(A_{f}, B_{f}+\epsilon P B_{s}\right)$ are controllable.

Based on information from (3), (7), and (10), we present in Figure 1 the block diagram for the reduced-order observerbased controller when only all slow state variables are perfectly measured. In (11) and (14) we have chosen the feedback gains for the eigenvalue assignment problem. However, any feedback gains $F_{1}$ and $F_{2}$ can be used to control the system and provide corresponding design requirements. 


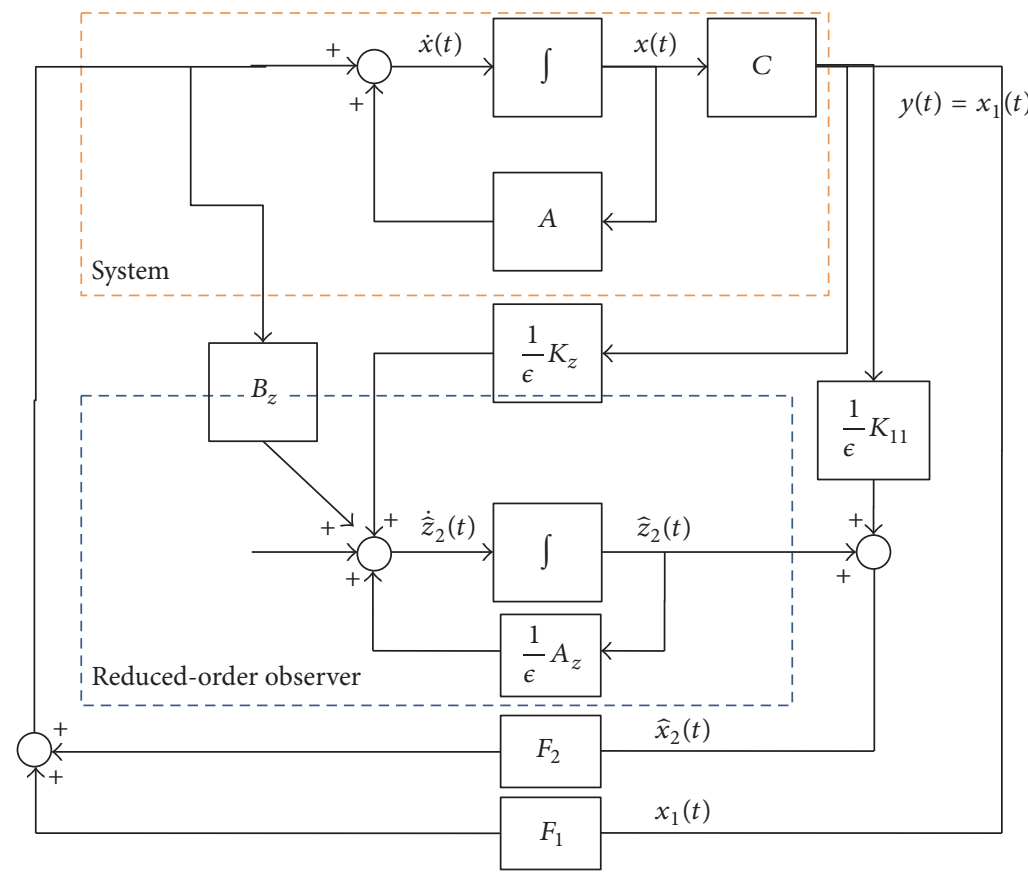

FIGURE 1: Case I: reduced-order observer-based controller design for a singularly perturbed linear system when only all slow state variables are directly measured.

\section{Case II: Controller Design When Only All Fast Variables Are Measured}

Consider the linear time invariant singularly perturbed control system [1], when only all fast state variable are directly measured:

$$
\begin{aligned}
\dot{x}_{1}(t) & =A_{11} x_{1}(t)+A_{12} x_{2}(t)+B_{1} u(t) \\
\dot{x}_{2}(t) & =\frac{1}{\epsilon} A_{21} x_{1}(t)+\frac{1}{\epsilon} A_{22} x_{2}(t)+\frac{1}{\epsilon} B_{2} u(t) \\
y(t) & =x_{2}(t) .
\end{aligned}
$$

The reduced-order observer for system (15) is given by [7]

$$
\dot{\widehat{z}}_{1}(t)=A_{z} \widehat{z}_{1}(t)+B_{z} u(t)+K_{z} y(t),
$$

where

$$
\begin{aligned}
& A_{z}=A_{11}-\frac{1}{\epsilon} K_{12} A_{21}, \\
& B_{z}=B_{2}-\frac{1}{\epsilon} K_{12} B_{1} \\
& K_{z}=A_{12}+A_{11} K_{12}-\frac{1}{\epsilon} K_{12} A_{22}-\frac{1}{\epsilon} K_{12} A_{21} K_{12} .
\end{aligned}
$$

The slow state estimation is obtained from

$$
\widehat{x}_{1}(t)=\widehat{z}_{1}(t)+K_{12} y(t)
$$

so that

$$
\widehat{x}(t)=\left[\begin{array}{l}
\hat{x}_{1}(t) \\
x_{2}(t)
\end{array}\right]=\left[\begin{array}{l}
\hat{x}_{1}(t) \\
y(t)
\end{array}\right] .
$$

The matrix $K_{12}$ is chosen to stabilize the reduced-order observer (16)-(17); that is,

$$
\begin{aligned}
\lambda\left(A_{11}-\frac{1}{\epsilon} K_{12} A_{21}\right) & =\lambda\left(A_{11}^{T}-\frac{1}{\epsilon} A_{21}^{T} K_{12}^{T}\right) \\
& =\lambda_{\text {robs }}^{\text {desired }} .
\end{aligned}
$$

The reduced-order observer (16) can be designed under the following assumption [14].

Assumption 4. The pair $\left(A_{11}^{T},(1 / \epsilon) A_{21}^{T}\right)$ is controllable, which is equivalent to the pair $\left(A_{11},(1 / \epsilon) A_{21}\right)$ being observable. It was shown in Appendix that this is equivalent to $\left(A_{11}, A_{21}\right)$ being observable.

Additional matrices needed in this design can be obtained from (9) and (12)-(13). Using the separation principle, the observer-based controller can be designed via the two-stage design as

$$
\begin{aligned}
u(t) & =-F x(t)=-\left[\begin{array}{ll}
F_{1} & F_{2}
\end{array}\right]\left[\begin{array}{l}
\widehat{x}_{1}(t) \\
x_{2}(t)
\end{array}\right] \\
& =-\left[\begin{array}{ll}
F_{s}+F_{f 2} P & F_{f 2}
\end{array}\right] T_{c}^{-1}\left[\begin{array}{c}
\widehat{x}_{1}(t) \\
y(t)
\end{array}\right],
\end{aligned}
$$

where $F_{s}$ and $F_{f 2}$ are obtained from (11) and (14). The feedback gains $F_{s}$ and $F_{f 2}$ can be obtained under Assumption 3.

In Figure 2, the block diagram for the reduced-order observer-based controller when only all fast variables are perfectly measured is presented. 


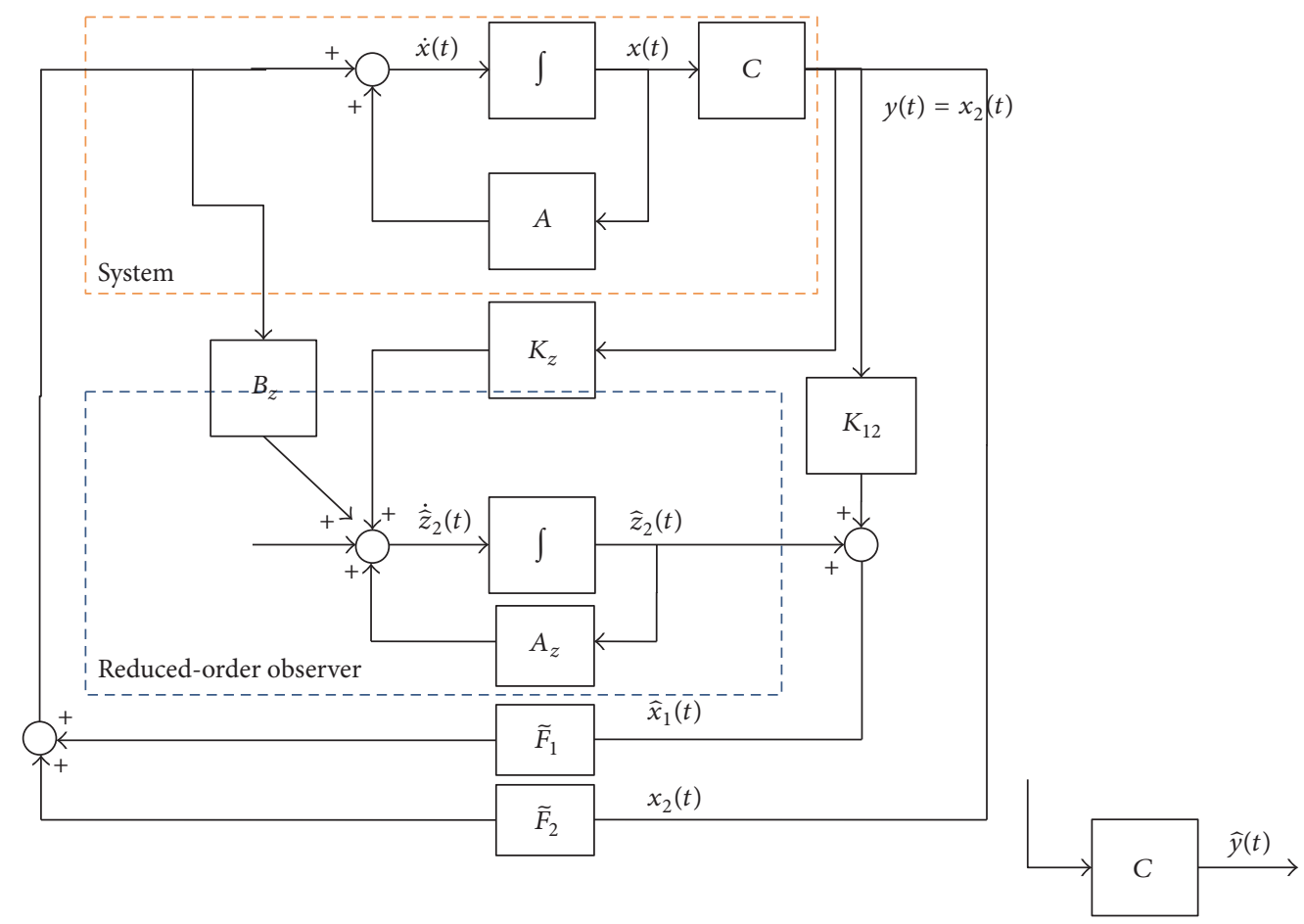

FIGURE 2: Case II: slow and fast observer-based controller design for a singularly perturbed linear system when only fast state variables are measured directly.

\section{Cases (III)-(V): Controller Design When Some Combinations of Slow and Fast Variables Are Measured}

In Case (III), the measurable states $x_{11}(t)$ are parts of the slow state vector $x_{1}(t)$ in the singularly perturbed linear system defined in (1), as

$$
\begin{aligned}
\dot{x}_{1}(t) & =A_{11} x_{1}(t)+A_{12} x_{2}(t) \\
\epsilon \dot{x}_{2}(t) & =A_{21} x_{1}(t)+A_{22} x_{2}(t) \\
y(t) & =x_{11}(t),
\end{aligned}
$$

where

$$
x_{1}(t)=\left[\begin{array}{l}
x_{11}(t) \\
x_{12}(t)
\end{array}\right]=\left[\begin{array}{c}
y(t) \\
x_{12}(t)
\end{array}\right] .
$$

Use the following partitioning:

$$
\begin{aligned}
& A_{11}=\left[\begin{array}{ll}
a_{11} & a_{12} \\
a_{21} & a_{22}
\end{array}\right], \\
& A_{12}=\left[\begin{array}{l}
a_{13} \\
a_{23}
\end{array}\right] \\
& A_{21}=\left[\begin{array}{ll}
a_{31} & a_{32}
\end{array}\right], \\
& A_{22}=\left[a_{33}\right],
\end{aligned}
$$

where dimensions of matrices $a_{11}, a_{22}$ and $a_{33}$ are, respectively, $a_{11} \in R^{l \times l}, a_{22} \in R^{\left(n_{1}-l\right) \times\left(n_{1}-l\right)}$, and $a_{33} \in R^{n_{2} \times n_{2}}$, with corresponding dimension of $a_{i j}, i \neq j$, matrices. System (22) with (23)-(24) can be represented as

$$
\begin{aligned}
\dot{x}_{11}(t) & =A_{11}^{r} x_{11}(t)+A_{12}^{r} x_{2}^{r}(t) \\
\dot{x}_{2}^{r}(t) & =A_{21}^{r} x_{11}(t)+A_{22}^{r} x_{2}^{r}(t) \\
y(t) & =x_{11}(t)
\end{aligned}
$$

where

$$
\begin{aligned}
x_{2}^{r}(t) & =\left[\begin{array}{l}
x_{12}(t) \\
x_{2}(t)
\end{array}\right] \\
A_{11}^{r} & =\left[a_{11}\right], \\
A_{12}^{r} & =\left[\begin{array}{ll}
a_{12} & a_{13}
\end{array}\right] \\
A_{21}^{r} & =\left[\begin{array}{l}
a_{21} \\
\frac{1}{\epsilon} a_{31}
\end{array}\right], \\
A_{22}^{r} & =\left[\begin{array}{ll}
a_{22} & a_{23} \\
\frac{1}{\epsilon} a_{32} & \frac{1}{\epsilon} a_{33}
\end{array}\right] .
\end{aligned}
$$


The reduced-order observer for this case is derived in [1], and it is given by

$$
\dot{\vec{z}}_{2}^{r}(t)=A_{z}^{r} \widehat{z}_{2}^{r}(t)+K_{z}^{r} y(t)
$$

where

$$
\begin{aligned}
A_{z}^{r} & =A_{22}^{r}-K_{2} A_{12}^{r} \\
& =\left[\begin{array}{cc}
a_{22} & a_{23} \\
\frac{1}{\epsilon} a_{32} & \frac{1}{\epsilon} a_{33}
\end{array}\right]-\left[\begin{array}{c}
K_{21} \\
K_{22}
\end{array}\right]\left[\begin{array}{ll}
a_{12} & a_{13}
\end{array}\right], \\
K_{z}^{r} & =A_{21}^{r}-K_{2} A_{11}^{r}+A_{22}^{r} K_{2}-K_{2} A_{12}^{r} K_{2}, \\
\widehat{z}_{2}^{r}(t) & =\left[\begin{array}{c}
\widehat{z}_{12}(t) \\
\widehat{z}_{2}(t)
\end{array}\right] .
\end{aligned}
$$

For the eigenvalue assignment in $A_{z}^{r}$, we encounter the singularly perturbed structure, so that the two-stage method is applied for a two time-scale problem.

The reduced-order sequential observer configuration obtained using the two-stage method [1] is given by

$$
\begin{aligned}
& {\left[\begin{array}{c}
\dot{\vec{z}}_{s}(t) \\
\epsilon \dot{\vec{z}}_{f \text { new }}(t)
\end{array}\right]} \\
& =\left[\begin{array}{cc}
A_{s r}-K_{s r} C_{s r} & 0 \\
-\epsilon K_{f 2 r} C_{s r} & A_{f r}-K_{f 2 r} C_{f \text { new } r}
\end{array}\right]\left[\begin{array}{c}
\widehat{z}_{s}(t) \\
\widehat{z}_{f \text { new }}(t)
\end{array}\right] \\
& +\left[\begin{array}{c}
K_{s r}^{*} \\
K_{f 2 r}^{*}
\end{array}\right] y(t),
\end{aligned}
$$

where $K_{s r}^{*}$ and $K_{f 2 r}^{*}$ are obtained from $T_{4 r}^{-1} K_{z}^{r}$, with $T_{4 r}$ defined by

$$
\left[\begin{array}{c}
\widehat{z}_{12}(t) \\
\widehat{z}_{2}(t)
\end{array}\right]=T_{1 r} T_{c r} T_{2 r}=T_{4 r}\left[\begin{array}{c}
\widehat{z}_{s}(t) \\
\widehat{z}_{f \text { new }}(t)
\end{array}\right],
$$

where

$$
T_{1 r}=\left[\begin{array}{cc}
I_{\left(n_{1}-l\right)} & 0 \\
0 & \frac{1}{\epsilon} I_{n_{2}}
\end{array}\right],
$$

$$
\begin{aligned}
& T_{2 r}=\left[\begin{array}{cc}
I_{\left(n_{1}-l\right)} & P_{o r}^{T} \\
0 & I_{n_{2}}
\end{array}\right], \\
& T_{c r}^{T}=\left[\begin{array}{cc}
I_{\left(n_{1}-l\right)} & -\epsilon L_{r}^{T} \\
H_{r}^{T} & I_{n_{2}}-\epsilon H_{r}^{T} L_{r}^{T}
\end{array}\right],
\end{aligned}
$$

where $L_{r}^{T}$ and $H_{r}^{T}$ are matrices that satisfy

$$
\begin{aligned}
& 0=\epsilon\left(a_{22}^{T}-L_{r}^{T} a_{23}^{T}\right) L_{r}^{T}+\left(a_{32}^{T}-L_{r}^{T} a_{33}^{T}\right) \\
& 0=\epsilon H_{r}^{T}\left(a_{22}^{T}-L_{r}^{T} a_{23}^{T}\right)+a_{23}^{T}-\left(a_{33}^{T}+\epsilon a_{23}^{T} L_{r}^{T}\right) H_{r}^{T}
\end{aligned}
$$

with $a_{i j}$ matrices defined in (26). The reduced-order observer (29) has a sequential structure. It can be block diagonalized and used in a parallel structure as follows:

$$
\begin{aligned}
\dot{\vec{z}}_{s}(t)= & \left(A_{s r}-K_{s r} C_{s r}\right) \widehat{z}_{s}(t)+K_{s r}^{*} y(t) \\
\epsilon \dot{\vec{z}}_{f \text { new } 2}(t)= & \left(A_{f r}-K_{f 2 r} C_{f \text { new } r}\right) \widehat{z}_{f \text { new } 2}(t) \\
& +K_{f 3 r} y(t),
\end{aligned}
$$

where

$$
\begin{aligned}
K_{f 3 r} & =\epsilon\left(P_{o 2 r} K_{s r}^{*}+K_{f 2 r}^{*}\right), \\
{\left[\begin{array}{c}
\widehat{z}_{s}(t) \\
\widehat{z}_{f \text { new } 2}(t)
\end{array}\right] } & =\left[\begin{array}{cc}
I_{\left(n_{1}-l\right)} & 0 \\
P_{o 2 r} & I_{n_{2}}
\end{array}\right]\left[\begin{array}{c}
\widehat{z}_{s}(t) \\
\widehat{z}_{f \text { new }}(t)
\end{array}\right] \\
& =T_{3 r}\left[\begin{array}{c}
\widehat{z}_{s}(t) \\
\widehat{z}_{f \text { new }}(t)
\end{array}\right] .
\end{aligned}
$$

The original coordinates $\widehat{z}_{12}(t)$ and $\widehat{z}_{2}(t)$ and the coordinates $\widehat{z}_{s}(t)$ and $\widehat{z}_{\text {fnew } 2}(t)$ are related via

$$
\begin{aligned}
{\left[\begin{array}{c}
\widehat{z}_{12}(t) \\
\widehat{z}_{2}(t)
\end{array}\right]=} & T_{4 r} T_{3 r}^{-1}\left[\begin{array}{c}
\widehat{z}_{s}(t) \\
\widehat{z}_{f \text { new } 2}(t)
\end{array}\right]=T_{r}\left[\begin{array}{c}
\widehat{z}_{s}(t) \\
\widehat{z}_{f \text { new } 2}(t)
\end{array}\right] \\
& =\left[\begin{array}{cc}
I_{\left(n_{1}-l\right)}-P_{o r}^{T} P_{o 2 r}-H_{r} P_{o 2 r} & P_{o r}^{T}+H_{r} \\
-L_{r}+L_{r} P_{o r}^{T} P_{o 2 r}-\frac{1}{\epsilon}\left(I_{n_{2}}-\epsilon L_{r} H_{r}\right) P_{o 2 r} & -L_{r} P_{o r}^{T}+\frac{1}{\epsilon}\left(I_{n_{2}}-\epsilon L_{r} H_{r}\right)
\end{array}\right]\left[\begin{array}{c}
\widehat{z}_{s}(t) \\
\widehat{z}_{f \text { new2 }}(t)
\end{array}\right],
\end{aligned}
$$

where $P_{o 2 r}$ satisfies the algebraic Sylvester equation represented by

$$
\begin{aligned}
& \epsilon P_{o 2 r}\left(A_{s r}-K_{s r} C_{s r}\right)-\epsilon K_{f 2 r} C_{s r} \\
& \quad-\left(A_{f r}-K_{f 2 r} C_{f \text { new } r}\right) P_{o 2 r}=0 \Longrightarrow
\end{aligned}
$$

$$
P_{o 2 r}^{0}=O(\epsilon)
$$

The steps used in the sequential and parallel observer design structures are summarized in Figure 3. 


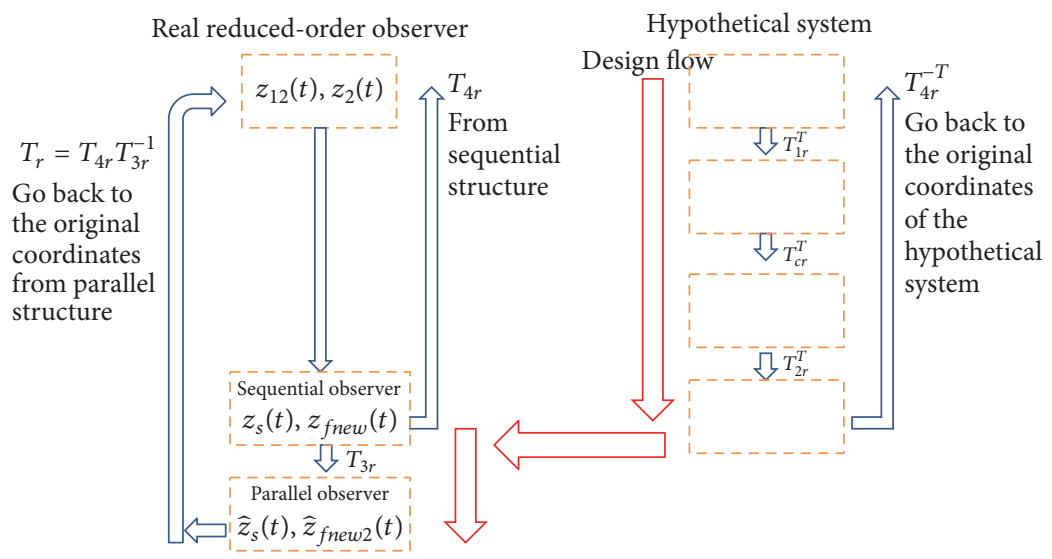

FIgURE 3: Case III: design outlines for the sequential and parallel reduced-order observer.

We use the parallel observers structure (33) and consider the reduced-order observer-based controller design for singularly perturbed linear systems. Observer (33) is now driven by the system measurements and control inputs; that is,

$$
\begin{aligned}
\dot{\vec{z}}_{s}(t)= & \left(A_{s r}-K_{s r} C_{s r}\right) \widehat{z}_{s}(t)+B_{s r 2} u(t) \\
& +K_{s r}^{*} y(t) \\
\epsilon \dot{\vec{z}}_{f \text { new } 2}(t)= & \left(A_{f q}-K_{f 2} C_{f \text { new }}\right) \widehat{z}_{f \text { new } 2}(t) \\
& +B_{f 2 r} u(t)+K_{f 3} y(t),
\end{aligned}
$$

where $B_{s r 2}$ and $B_{f 2 r}$ can be obtained from $T_{r}^{-1} B$ as

$$
\begin{aligned}
B_{s r 2}= & \left(I_{\left(n_{1}-l\right)}-\epsilon H_{r} L_{r}\right) B_{z 1}-\epsilon P_{o r}^{T} L_{r} B_{z 1}-H_{r} B_{z 2} \\
& -P_{o r}^{T} B_{z 2}, \\
B_{f 2 r}= & \epsilon P_{o 2 r}\left(I_{\left(n_{1}-l\right)}-\epsilon H_{r} L_{r}\right) B_{z 1}-\epsilon^{2} P_{o 2 r} P_{o r}^{T} L_{r} B_{z 1} \\
& +\epsilon^{2} L_{r} B_{z 1}-\epsilon P_{o 2 r} H_{r} B_{z 2}-\epsilon P_{o 2 r} P_{o r}^{T} B_{z 2} \\
& +\epsilon B_{z 2} .
\end{aligned}
$$

The control input in the $\widehat{z}_{s}-\widehat{z}_{f \text { new } 2}$ coordinates is given by

$$
\begin{aligned}
& u(t)=-F_{r} \hat{x}(t)=-\left[\begin{array}{ll}
F_{12} & F_{2}
\end{array}\right]\left[\begin{array}{c}
\hat{x}_{12}(t) \\
\hat{x}_{2}(t)
\end{array}\right] \\
& =-\left(\left[\begin{array}{ll}
F_{12} & F_{2}
\end{array}\right]\left[\begin{array}{l}
K_{21} \\
K_{22}
\end{array}\right] y(t)\right. \\
& \left.+\left[\begin{array}{ll}
F_{12} & F_{2}
\end{array}\right] T_{r}\left[\begin{array}{c}
\widehat{z}_{s}(t) \\
\widehat{z}_{\text {fnew } 2}(t)
\end{array}\right]\right) \\
& =-F_{r} K_{2} y(t)-F_{s r 2} \widehat{z}_{s}(t)-F_{f r 2} \widehat{z}_{f \text { new } 2}(t), \\
& {\left[\begin{array}{llll}
F_{11} & F_{12} & F_{2}
\end{array}\right]=\left[\begin{array}{lll}
F_{1} & \mid F_{2}
\end{array}\right]} \\
& =\left[\begin{array}{lll}
F_{s}+F_{f 2} P & \mid F_{f 2}
\end{array}\right] T_{c}^{-1}
\end{aligned}
$$

so that $\left[F_{11} \mid F_{12} F_{2}\right]=\left[\begin{array}{lll}F_{11} & \mid F_{r}\end{array}\right]$ with

$$
\begin{aligned}
F_{s r 2}= & F_{12}\left(I_{\left(n_{1}-l\right)}-P_{o r}^{T} P_{o 2 r}-H_{r} P_{o 2 r}\right) \\
& -F_{2} L\left(I_{n}-P_{o r}^{T} P_{o 2 r}\right) \\
& -\frac{1}{\epsilon} F_{2}\left(I_{n_{2}}-\epsilon L_{r} H_{r}\right) P_{o 2 r} \\
F_{f r 2}= & F_{12}\left(P_{o r}^{T}+H_{r}\right)-F_{2} L_{r} P_{o r}^{T} \\
& +\frac{1}{\epsilon} F_{2}\left(I_{n_{2}}-\epsilon L_{r} H_{r}\right) .
\end{aligned}
$$

The corresponding block diagram for the observer driven controller is presented in Figure 4. This block diagram clearly indicates full parallelism of the slow controller driven by the slow observer and the fast controller driven by the fast observer.

The remaining matrices defined in (29) are given by

$$
\begin{aligned}
A_{s r}^{T} & =a_{22}^{T}-L^{T} a_{23}^{T}, \\
A_{f r}^{T} & =a_{33}^{T}+\epsilon a_{23}^{T} L^{T} \\
C_{s r}^{T} & =a_{12}^{T}-L^{T} a_{13}^{T}, \\
C_{f r}^{T} & =\epsilon H^{T} a_{12}^{T}+\left(I_{n_{2}}-\epsilon H^{T} L^{T}\right) a_{13}^{T} \\
C_{f \text { new } r} & =C_{f r}+\epsilon C_{s r} P_{o r}^{T} .
\end{aligned}
$$

$K_{s r}, K_{f 2 r}$, and $P_{o r}$ can be obtained from the following formulas:

$$
\begin{aligned}
& \lambda\left(A_{s r}^{T}-C_{s r}^{T} K_{s r}^{T}\right)=\lambda\left(A_{s r}-K_{s r} C_{s r}\right)=\lambda_{s}^{\text {desired }} \\
& \lambda\left(A_{f r}-K_{f 2 r} C_{f \text { new } r}\right)=\lambda_{f}^{\text {desired }} \\
& \epsilon P_{o r}\left(A_{s r}^{T}-C_{s r}^{T} K_{s r}^{T}\right)-C_{f r}^{T} K_{s r}^{T}-A_{f r}^{T} P_{o r}=0 \Longrightarrow \\
& P_{o r}=O(\epsilon) .
\end{aligned}
$$




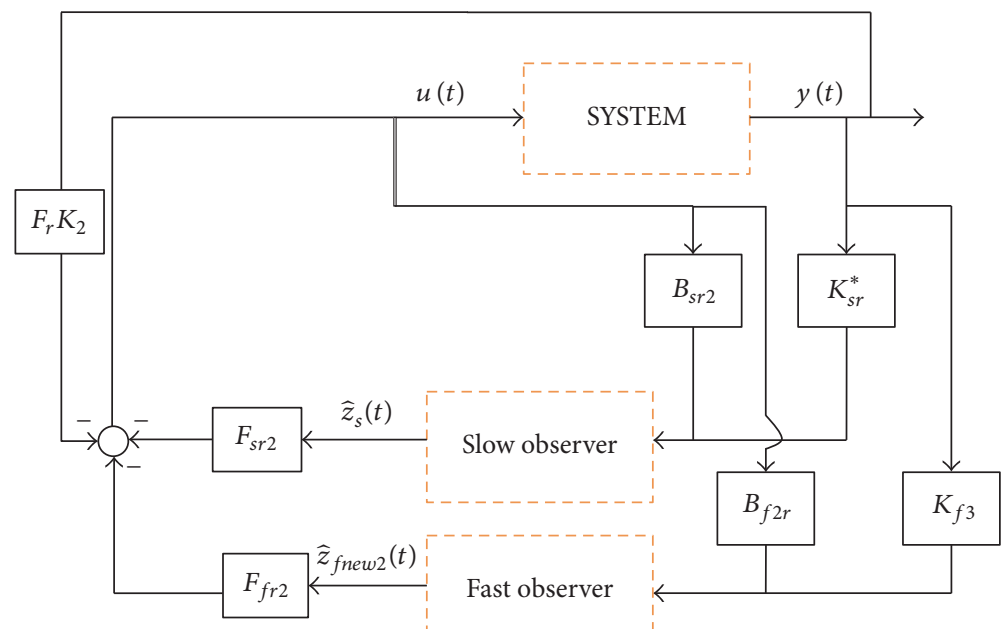

FiguRE 4: Case III: slow and fast observer-based controller designs for singularly perturbed linear systems with the system feedback gains obtained in (39) when parts of slow variables are measured.

In Case (IV), the measurable states $x_{21}(t)$ are parts of the fast state vector $x_{2}(t)$ in the singularly perturbed linear system defined in (1):

$$
\begin{aligned}
\dot{x}_{1}(t) & =A_{11} x_{1}(t)+A_{12} x_{2}(t) \\
\epsilon \dot{x}_{2}(t) & =A_{21} x_{1}(t)+A_{22} x_{2}(t) \\
y(t) & =I_{1} x_{22}(t),
\end{aligned}
$$

where

$$
x_{2}(t)=\left[\begin{array}{l}
x_{21}(t) \\
x_{22}(t)
\end{array}\right]=\left[\begin{array}{c}
x_{21}(t) \\
y(t)
\end{array}\right]
$$

using the following partitioning:

$$
\begin{aligned}
& A_{11}=\left[a_{11}\right], \\
& A_{12}=\left[\begin{array}{ll}
a_{12} & a_{13}
\end{array}\right] \\
& A_{21}=\left[\begin{array}{l}
a_{21} \\
a_{31}
\end{array}\right], \\
& A_{22}=\left[\begin{array}{ll}
a_{22} & a_{23} \\
a_{32} & a_{33}
\end{array}\right],
\end{aligned}
$$

where dimensions of matrices $a_{11}, a_{22}$, and $a_{33}$ are, respectively, $a_{11} \in R^{n_{1} \times n_{1}}, a_{22} \in R^{\left(n_{2}-l\right) \times\left(n_{2}-l\right)}$, and $a_{33} \in R^{l \times l}$, with the corresponding dimensions of $a_{i j}, i \neq j$, matrices. System (43) with (44)-(45) can be represented as

$$
\begin{aligned}
\dot{x}_{1}^{r}(t) & =A_{11}^{r} x_{1}^{r}(t)+A_{12}^{r} x_{22}(t) \\
\dot{x}_{22}(t) & =A_{21}^{r} x_{1}^{r}(t)+A_{22}^{r} x_{22}(t) \\
y(t) & =I_{l} x_{22}(t),
\end{aligned}
$$

where

$$
\begin{aligned}
& x_{1}^{r}(t)=\left[\begin{array}{l}
x_{1}(t) \\
x_{21}(t)
\end{array}\right] \\
& A_{11}^{r}=\left[\begin{array}{cc}
a_{11} & a_{12} \\
\frac{1}{\epsilon} a_{21} & \frac{1}{\epsilon} a_{22}
\end{array}\right] \text {, } \\
& A_{12}^{r}=\left[\begin{array}{l}
a_{13} \\
\frac{1}{\epsilon} a_{23}
\end{array}\right] \\
& A_{21}^{r}=\left[\begin{array}{ll}
\frac{1}{\epsilon} a_{31} & \frac{1}{\epsilon} a_{32}
\end{array}\right] \text {, } \\
& A_{22}^{r}=\left[\frac{1}{\epsilon} a_{33}\right] \text {. }
\end{aligned}
$$

The corresponding reduced-order observer structure is given by [1]

$$
\dot{\bar{z}}_{1}^{r}(t)=A_{z, 2}^{r} \widehat{z}_{1}^{r}(t)+B_{z, 2}^{r} u(t)+K_{z, 2}^{r} y(t)
$$

where

$$
\widehat{z}_{1}^{r}(t)=\left[\begin{array}{c}
\widehat{z}_{1}(t) \\
\widehat{z}_{21}(t)
\end{array}\right]
$$

The observer gain $K_{3}$ and matrices $B_{z, 2}^{r}$ and $K_{z, 2}^{r}$ [7] are obtained from

$$
\begin{aligned}
A_{z, 2}^{r} & =A_{11}^{r}-K_{3} A_{21}^{r} \\
& =\left[\begin{array}{ll}
a_{11} & a_{12} \\
\frac{1}{\epsilon} a_{21} & \frac{1}{\epsilon} a_{22}
\end{array}\right]-\left[\begin{array}{l}
K_{31} \\
K_{32}
\end{array}\right]\left[\begin{array}{ll}
\frac{1}{\epsilon} a_{31} & \frac{1}{\epsilon} a_{32}
\end{array}\right],
\end{aligned}
$$




$$
\begin{aligned}
& B_{z, 2}^{r}=B_{2}-K_{3} B_{1}=\left[\begin{array}{l}
B_{z 1} \\
B_{z 2}
\end{array}\right], \\
& K_{z, 2}^{r}=A_{12}^{r}-K_{3} A_{22}^{r}+A_{11}^{r} K_{3}-K_{3} A_{21}^{r} K_{3} .
\end{aligned}
$$

For the eigenvalue assignment in $A_{z, 2}^{r}$, we encounter singularly perturbed structure so that the two-stage design is applied to the slow and fast subsystems.

The two-stage method provides the parallel observer [1]. Using the parallel observer we can form the reduced-order observer-based controller given as

$$
\begin{aligned}
& {\left[\begin{array}{c}
\dot{\bar{z}}_{s, 2}(t) \\
\epsilon \dot{\bar{z}}_{f \text { new } 2,2}(t)
\end{array}\right]} \\
& =\left[\begin{array}{cc}
A_{s r, 2}-K_{s r, 2} C_{s r, 2} & 0 \\
0 & A_{f r, 2}-K_{f 2 r, 2} C_{f \text { new } r, 2}
\end{array}\right]\left[\begin{array}{c}
\widehat{z}_{s, 2}(t) \\
\widehat{z}_{f \text { new } 2,2}(t)
\end{array}\right] \\
& +\left[\begin{array}{c}
B_{s 2 r, 2} \\
B_{f 2 r, 2}
\end{array}\right] u(t)+\left[\begin{array}{c}
K_{s r, 2}^{*} \\
K_{f 3 r, 2}
\end{array}\right] y(t),
\end{aligned}
$$

where

$$
\begin{aligned}
& A_{s r, 2}^{T}=a_{11}^{T}-L_{r, 2}^{T} a_{12}^{T}, \\
& A_{f r, 2}^{T}=a_{22}^{T}+\epsilon a_{12}^{T} L_{r, 2}^{T} \\
& B_{s 2 r, 2}=\left(I_{n_{1}}-\epsilon H_{r, 2} L_{r, 2}\right) B_{z 1}-\epsilon P_{o r, 2}^{T} L_{r, 2} B_{z 1}
\end{aligned}
$$$$
\left[\begin{array}{c}
\widehat{z}_{1}(t) \\
\widehat{z}_{21}(t)
\end{array}\right]=T_{r, 2}\left[\begin{array}{c}
\widehat{z}_{s, 2}(t) \\
\widehat{z}_{f \text { new }, 2}(t)
\end{array}\right]=T_{4 r, 2} T_{3 r, 2}^{-1}\left[\begin{array}{c}
\widehat{z}_{s, 2}(t) \\
\widehat{z}_{f \text { new }, 2}(t)
\end{array}\right]
$$$$
=\left[\begin{array}{cc}
I_{n_{1}}-P_{o r, 2}^{T} P_{o 2 r, 2}-H_{r, 2} P_{o 2 r, 2} & P_{o r, 2}^{T}+H_{r, 2} \\
-L_{r, 2}+L_{r, 2} P_{o r, 2}^{T} P_{o 2 r, 2}-\frac{1}{\epsilon}\left(I_{\left(n_{2}-l\right)}-\epsilon L_{r, 2} H_{r, 2}\right) P_{o 2 r, 2} & -L_{r, 2} P_{o r, 2}^{T}+\frac{1}{\epsilon}\left(I_{\left(n_{2}-l\right)}-\epsilon L_{r, 2} H_{r, 2}\right)
\end{array}\right]\left[\begin{array}{c}
\widehat{z}_{s, 2}(t) \\
\widehat{z}_{f \text { new } 2,2}(t)
\end{array}\right],
$$

and $L_{r, 2}^{T}$ and $H_{r, 2}^{T}$ are the solution given as

$$
\begin{aligned}
0= & \epsilon\left(a_{22}^{T}-L_{r, 2}^{T} a_{23}^{T}\right) L_{r, 2}^{T}+\left(a_{32}^{T}-L_{r, 2}^{T} a_{33}^{T}\right) \\
0= & \epsilon H_{r, 2}^{T}\left(a_{22}^{T}-L_{r, 2}^{T} a_{23}^{T}\right)+a_{23}^{T} \\
& -\left(a_{33}^{T}+\epsilon a_{23}^{T} L_{r, 2}^{T}\right) H_{r, 2}^{T} .
\end{aligned}
$$

The coordinates of the original observer (48) and the parallel observer (51) are related via a transformation as where

$$
\begin{aligned}
T_{4 r, 2} & =\left[\begin{array}{cc}
I_{n_{1}} & P_{o r, 2}^{T}+H_{r, 2} \\
-L_{r, 2} & -L_{r, 2} P_{o r, 2}^{T}+\frac{1}{\epsilon}\left(I_{\left(n_{2}-l\right)}-\epsilon L_{r, 2} H_{r, 2}\right)
\end{array}\right], \\
T_{3 r, 2}^{-1} & =\left[\begin{array}{cc}
I_{n_{1}} & 0 \\
-P_{o 2 r, 2} & I_{\left(n_{2}-l\right)}
\end{array}\right] .
\end{aligned}
$$

$K_{s r, 2}^{*}$ and $(1 / \epsilon) K_{f 2 r, 2}^{*}$ are obtained from $T_{4 r, 2}^{-1} K_{z, 2}^{r}{ }^{r}$. The slow feedback gain $K_{s r, 2}$ can be obtained using the eigenvalue assignment; that is,

$$
\begin{aligned}
\lambda\left(A_{s r, 2}^{T}-C_{s r, 2}^{T} K_{s r, 2}^{T}\right) & =\lambda\left(A_{s r, 2}-K_{s r, 2} C_{s r, 2}\right) \\
& =\lambda_{s}^{\text {desired }}
\end{aligned}
$$

requiring that the following assumption is satisfied [14].
Assumption 5. The pair $\left(A_{s r, 2}, C_{s r, 2}\right)$ is observable.

The fast feedback gain $K_{f 2 r, 2}$ is obtained from the eigenvalue assignment problem; that is,

$$
\lambda\left(A_{f r, 2}-K_{f 2 r, 2} C_{f \text { new } r, 2}\right)=\lambda_{f}^{\text {desired }} .
$$

The following observability assumption is needed [14].

Assumption 6. The pair $\left(A_{f r, 2}, C_{f \text { new } r, 2}\right)$ is observable.

The control input in $\widehat{z}_{s, 2}-\widehat{z}_{f \text { new2,2 }}$ coordinates is given by

$$
\begin{aligned}
u(t) & =-F_{r} \hat{x}(t)=-\left[\begin{array}{ll}
F_{1} & F_{21}
\end{array}\right]\left[\begin{array}{c}
\widehat{x}_{1}(t) \\
\widehat{x}_{21}(t)
\end{array}\right] \\
& =-F_{r, 2} K_{3} y(t)-\left[\begin{array}{ll}
F_{s r 2,2} & F_{f r 2,2}
\end{array}\right]\left[\begin{array}{c}
\widehat{z}_{s, 2}(t) \\
\widehat{z}_{f \text { new } 2,2}(t)
\end{array}\right]
\end{aligned}
$$




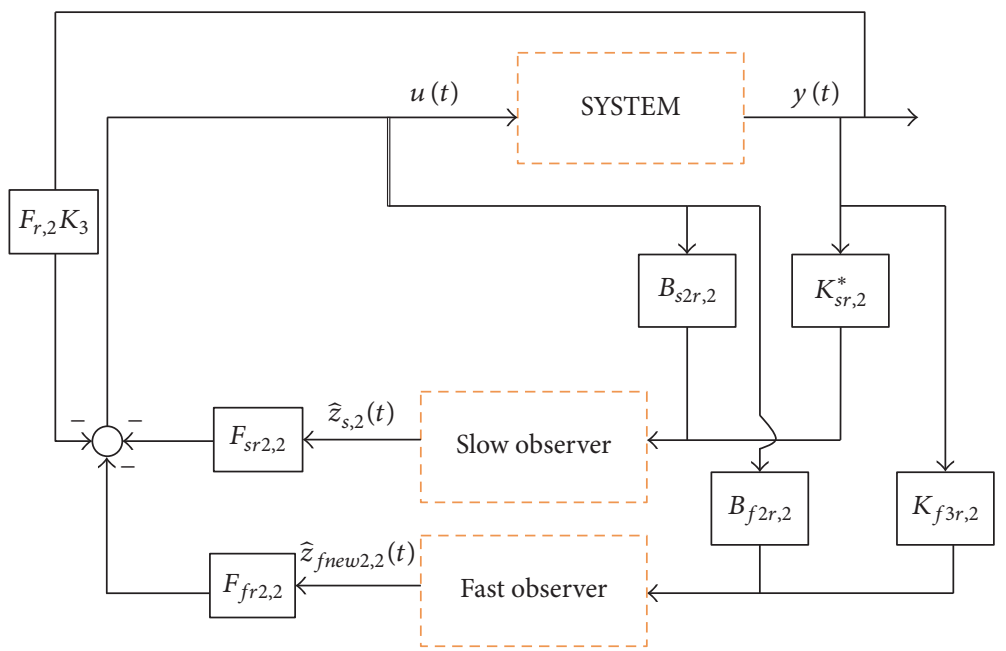

FIGURE 5: Case IV: slow and fast observer-based controller design for a singularly perturbed linear system measurable variables being part of the fast variables only.

$$
\left[\begin{array}{llll}
F_{1} & \mid F_{21} & F_{22}
\end{array}\right]=\left[\begin{array}{ll}
F_{1} & \mid F_{2}
\end{array}\right]=\left[F_{s}+F_{f 2} P \mid F_{f 2}\right] T_{c}^{-1}
$$

so that $\left[\begin{array}{lll}F_{1} & F_{21} & F_{22}\end{array}\right]=\left[F_{r} \mid F_{22}\right]$

with

$$
\begin{aligned}
F_{s r 2,2}= & F_{1}\left(I_{n_{1}}-P_{o r, 2}^{T} P_{o 2 r, 2}-H_{r, 2} P_{o 2 r, 2}\right) \\
& -F_{21} L\left(I_{\left(n_{2}-l\right)}-P_{o r, 2}^{T} P_{o 2 r, 2}\right) \\
& -\frac{1}{\epsilon} F_{2}\left(I_{\left(n_{2}-l\right)}-\epsilon L_{r, 2} H_{r, 2}\right) P_{o 2 r, 2} \\
F_{f r 2,2}= & F_{1}\left(P_{o r, 2}^{T}+H_{r, 2}\right)-F_{21} L_{r, 2} P_{o r, 2}^{T} \\
& +\frac{1}{\epsilon} F_{21}\left(I_{\left(n_{2}-l\right)}-\epsilon L_{r, 2} H_{r, 2}\right) .
\end{aligned}
$$

The remaining matrices $P_{o r, 2}$ and $P_{o 2 r, 2}$ satisfy the algebraic Sylvester equation represented by

$$
\begin{aligned}
& \epsilon P_{o r, 2}\left(A_{s r, 2}^{T}-C_{s r, 2}^{T} K_{s r, 2}^{T}\right)-C_{f r, 2}^{T} K_{s r, 2}^{T}-A_{f r, 2}^{T} P_{o r, 2} \\
& \quad=0 \Longrightarrow \\
& P_{o r, 2}=O(\epsilon) \\
& \epsilon P_{o 2 r, 2}\left(A_{s r, 2}-K_{s r, 2} C_{s r, 2}\right)-\epsilon K_{f 2 r, 2} C_{s r, 2} \\
& \quad-\left(A_{f r, 2}-K_{f 2 r, 2} C_{f \text { new } r, 2}\right) P_{o 2 r, 2}=0 \Longrightarrow \\
& P_{o 2 r, 2}^{0}=O(\epsilon) .
\end{aligned}
$$

The corresponding block diagram for the observer driven controller is presented in Figure 5.
In Case $(\mathrm{V})$, the measurable states $x_{11}(t)$ and $x_{21}(t)$ are parts of the slow state vector $x_{1}(t)$ and the fast state $x_{2}(t)$ in the singularly perturbed linear system defined as

$$
\begin{aligned}
\dot{x}_{1}(t) & =A_{11} x_{1}(t)+A_{12} x_{2}(t) \\
\epsilon \dot{x}_{2}(t) & =A_{21} x_{1}(t)+A_{22} x_{2}(t) \\
y(t) & =\left[\begin{array}{llll}
I & 0 & 0 & 0 \\
0 & 0 & I & 0
\end{array}\right]\left[\begin{array}{l}
x_{11}(t) \\
x_{12}(t) \\
x_{21}(t) \\
x_{22}(t)
\end{array}\right]=\left[\begin{array}{l}
x_{11}(t) \\
x_{21}(t)
\end{array}\right] \\
& =\left[\begin{array}{l}
y_{1}(t) \\
y_{2}(t)
\end{array}\right],
\end{aligned}
$$

where

$$
\begin{aligned}
& x_{1}(t)=\left[\begin{array}{l}
x_{11}(t) \\
x_{12}(t)
\end{array}\right], \\
& x_{1}(t)=\left[\begin{array}{l}
x_{11}(t) \\
x_{12}(t)
\end{array}\right]=\left[\begin{array}{l}
y_{1}(t) \\
x_{12}(t)
\end{array}\right], \\
& x_{2}(t)=\left[\begin{array}{l}
x_{21}(t) \\
x_{22}(t)
\end{array}\right]=\left[\begin{array}{l}
x_{21}(t) \\
y_{2}(t)
\end{array}\right],
\end{aligned}
$$

using the following partitioning:

$$
\begin{aligned}
& A_{11}=\left[\begin{array}{ll}
a_{11}^{*} & a_{12}^{*} \\
a_{21}^{*} & a_{22}^{*}
\end{array}\right], \\
& A_{12}=\left[\begin{array}{ll}
a_{13}^{*} & a_{14}^{*} \\
a_{23}^{*} & a_{24}^{*}
\end{array}\right]
\end{aligned}
$$




$$
\begin{aligned}
& A_{21}=\left[\begin{array}{ll}
a_{31}^{*} & a_{32}^{*} \\
a_{41}^{*} & a_{42}^{*}
\end{array}\right], \\
& A_{22}=\left[\begin{array}{ll}
a_{33}^{*} & a_{34}^{*} \\
a_{43}^{*} & a_{44}^{*}
\end{array}\right],
\end{aligned}
$$

where dimensions of matrices $a_{11}^{*}, a_{22}^{*}$, and $a_{33}^{*}$ are, respectively, $a_{11}^{*} \in R^{l_{1} \times l_{1}}, a_{22}^{*} \in R^{\left(n_{1}-l_{1}\right) \times\left(n_{1}-l_{1}\right)}$, and $a_{33}^{*} \in R^{l_{2} \times l_{2}}$, with the corresponding dimensions of $a_{i j}, i \neq j$, matrices. System (61) with (62)-(63) can be represented as

$$
\begin{aligned}
\dot{x}_{m}(t) & =A_{1}^{r} x_{m}(t)+A_{2}^{r} x_{u}(t) \\
\dot{x}_{u}(t) & =A_{3}^{r} x_{m}(t)+A_{4}^{r} x_{u}(t) \\
y(t) & =\left[\begin{array}{llll}
I & 0 & 0 & 0 \\
0 & 0 & I & 0
\end{array}\right]\left[\begin{array}{l}
x_{11}(t) \\
x_{12}(t) \\
x_{21}(t) \\
x_{22}(t)
\end{array}\right]=\left[\begin{array}{l}
x_{11}(t) \\
x_{21}(t)
\end{array}\right] \\
& =\left[\begin{array}{l}
y_{1}(t) \\
y_{2}(t)
\end{array}\right],
\end{aligned}
$$

where

$$
\begin{aligned}
x_{m}(t)= & {\left[\begin{array}{l}
x_{11}(t) \\
x_{21}(t)
\end{array}\right], } \\
x_{u}(t) & =\left[\begin{array}{l}
x_{12}(t) \\
x_{22}(t)
\end{array}\right] \\
A_{1}^{r} & =\left[\begin{array}{ll}
a_{11}^{*} & a_{13}^{*} \\
\frac{1}{\epsilon} a_{31}^{*} & \frac{1}{\epsilon} a_{33}^{*}
\end{array}\right], \\
A_{2}^{r} & =\left[\begin{array}{cc}
a_{12}^{*} & a_{14}^{*} \\
\frac{1}{\epsilon} a_{32}^{*} & \frac{1}{\epsilon} a_{34}^{*}
\end{array}\right] \\
A_{3}^{r}= & {\left[\begin{array}{cc}
a_{21}^{*} & a_{23}^{*} \\
\frac{1}{\epsilon} a_{41}^{*} & \frac{1}{\epsilon} a_{43}^{*}
\end{array}\right], } \\
A_{4}^{r}= & {\left[\begin{array}{cc}
a_{22}^{*} & a_{24}^{*} \\
\frac{1}{\epsilon} a_{42}^{*} & \frac{1}{\epsilon} a_{44}^{*}
\end{array}\right] . }
\end{aligned}
$$

$x_{m}(t)$ are the measurable states and $x_{u}(t)$ are the unmeasurable states. $A_{1}^{r}$ and $A_{3}^{r}$ are elements in (62) relevant to the measurable states and $A_{2}^{r}$ and $A_{4}^{r}$ are elements in (62) relevant to the unmeasurable states. The reduced-order observer is given by

$$
\dot{\vec{z}}_{1}^{r}(t)=A_{z, 3}^{r} \widehat{z}_{1}^{r}(t)+B_{z, 3}^{r} u(t)+K_{z, 3}^{r} y(t) .
$$

The observer gains are obtained from

$$
\begin{aligned}
A_{z, 3}^{r} & =A_{4}^{r}-K_{4} A_{2}^{r} \\
& =\left[\begin{array}{cc}
a_{22}^{*} & a_{24}^{*} \\
\frac{1}{\epsilon} a_{42}^{*} & \frac{1}{\epsilon} a_{44}^{*}
\end{array}\right]-\left[\begin{array}{l}
K_{41} \\
K_{42}
\end{array}\right]\left[\begin{array}{cc}
a_{12}^{*} & a_{14}^{*} \\
\frac{1}{\epsilon} a_{32}^{*} & \frac{1}{\epsilon} a_{34}^{*}
\end{array}\right], \\
B_{z, 3}^{r} & =B_{2}-K_{4} B_{1}=\left[\begin{array}{l}
B_{z 1} \\
B_{z 2}
\end{array}\right], \\
K_{z, 3}^{r} & =A_{3}^{r}-K_{4} A_{1}^{r}+A_{4}^{r} K_{4}-K_{4} A_{2}^{r} K_{4} .
\end{aligned}
$$

For the eigenvalue assignment in $A_{z, 3}^{r}$, we encounter again the singularly perturbed structure.

The two-stage method provides the parallel observer; we can form the reduced-order observer-based controller given as [1]

$$
\begin{aligned}
& {\left[\begin{array}{c}
\dot{\vec{z}}_{s, 3}(t) \\
\epsilon \dot{\bar{z}}_{f \text { new } 2,3}(t)
\end{array}\right]} \\
& =\left[\begin{array}{cc}
A_{s r, 3}-K_{s r, 3} C_{s r, 3} & 0 \\
0 & A_{f r, 3}-K_{f 2 r, 3} C_{f \text { new } r, 3}
\end{array}\right]\left[\begin{array}{c}
\widehat{z}_{s, 3}(t) \\
\widehat{z}_{f \text { new } 2,3}(t)
\end{array}\right] \\
& +\left[\begin{array}{c}
B_{s 2 r, 3} \\
B_{f 2 r, 3}
\end{array}\right] u(t)+\left[\begin{array}{c}
K_{s r, 3}^{*} \\
K_{f 3 r, 3}
\end{array}\right] y(t),
\end{aligned}
$$

where

$$
\begin{aligned}
A_{s r, 3}^{T}= & a_{22}^{* T}-L_{r, 3}^{T} a_{24}^{* T}, \\
A_{f r, 3}^{T}= & a_{44}^{* T}+\epsilon a_{24}^{* T} L_{r, 3}^{T} \\
B_{s r 2,3}= & \left(I_{\left(n_{1}-l_{1}\right)}-\epsilon H_{r, 3} L_{r, 3}\right) B_{z 1}-\epsilon P_{o r, 3}^{T} L_{r, 3} B_{z 1} \\
& -H_{r, 3} B_{z 2}-P_{o r, 3}^{T} B_{z 2}, \\
B_{f 2 r, 3}= & \epsilon P_{o 2 r, 3}\left(I_{\left(n_{1}-l_{1}\right)}-\epsilon H_{r, 3} L_{r, 3}\right) B_{z 1} \\
& -\epsilon^{2} P_{o 2 r, 3} P_{o r, 3}^{T} L_{r, 3} B_{z 1}+\epsilon^{2} L_{r, 3} B_{z 1} \\
& -\epsilon P_{o 2 r, 3} H_{r, 3} B_{z 2}-\epsilon P_{o 2 r, 3} P_{o r, 3}^{T} B_{z 2} \\
& +\epsilon B_{z 2} P_{o 2 r, 2} P_{o r, 2}^{T} L_{r, 2} B_{z 1}+\epsilon^{2} L_{r, 2} B_{z 1} \\
& -\epsilon P_{o 2 r, 2} H_{r, 2} B_{z 2}-\epsilon P_{o 2 r, 2} P_{o r, 2}^{T} B_{z 2} \\
& +\epsilon B_{z 2} \\
C_{s r, 32}^{T}= & \epsilon a_{32}^{* T}-L_{r, 3}^{T} \frac{1}{\epsilon} a_{34}^{* T} \\
C_{s r, 31}^{T}= & a_{12}^{* T}-L_{r, 3}^{T} a_{14}^{* T}, \\
& 1 \\
C^{*} &
\end{aligned}
$$




$$
\begin{aligned}
C_{f r, 31}^{T} & =\epsilon H_{r, 3}^{T} a_{12}^{* T}+\left(I_{\left(n_{2}-l_{2}\right)}-\epsilon H_{r, 3}^{T} L_{r, 3}^{T}\right) a_{14}^{* T}, \\
C_{f r, 32}^{T} & =\epsilon H_{r, 3}^{T} \frac{1}{\epsilon} a_{32}^{* T}+\left(I_{\left(n_{2}-l_{2}\right)}-\epsilon H_{r, 3}^{T} L_{r, 3}^{T}\right) \frac{1}{\epsilon} a_{34}^{* T} \\
C_{s r, 3}^{T} & =\left[\begin{array}{ll}
C_{s r, 31}^{T} & C_{s r, 32}^{T}
\end{array}\right], \\
C_{f r, 3}^{T} & =\left[\begin{array}{ll}
C_{f r, 31}^{T} & C_{f r, 32}^{T}
\end{array}\right] \\
C_{f \text { new } r, 3} & =\left(C_{f r, 3}+\epsilon C_{s r, 3} P_{o r, 3}^{T}\right)
\end{aligned}
$$

and $L_{r, 3}^{T}$ and $H_{r, 3}^{T}$ are the solution given as

$$
\begin{aligned}
& 0=\epsilon\left(a_{22}^{* T}-L_{r, 3}^{T} a_{24}^{* T}\right) L_{r, 3}^{T}+\left(a_{42}^{* T}-L_{r, 3}^{T} a_{44}^{* T}\right) \\
& 0=\epsilon H_{r, 3}^{T}\left(a_{22}^{* T}-L_{r, 3}^{T} a_{24}^{* T}\right)+a_{24}^{* T}
\end{aligned}
$$

$$
\begin{aligned}
& {\left[\begin{array}{c}
\widehat{z}_{12}(t) \\
\widehat{z}_{22}(t)
\end{array}\right]=T_{r, 3}\left[\begin{array}{c}
\widehat{z}_{s, 3}(t) \\
\widehat{z}_{f \text { new } 2,3}(t)
\end{array}\right]=T_{4 r, 3} T_{3 r, 3}^{-1}\left[\begin{array}{c}
\widehat{z}_{s, 3}(t) \\
\widehat{z}_{f \text { new } 2,3}(t)
\end{array}\right]} \\
& =\left[\begin{array}{cc}
I_{\left(n_{1}-l_{1}\right)}-P_{o r, 3}^{T} P_{o 2 r, 3}-H_{r, 3} P_{o 2 r, 3} & P_{o r, 3}^{T}+H_{r, 3} \\
-L_{r, 3}+L_{r, 3} P_{o r, 3}^{T} P_{o 2 r, 3}-\frac{1}{\epsilon}\left(I_{\left(n_{2}-l_{2}\right)}-\epsilon L_{r, 3} H_{r, 3}\right) P_{o 2 r, 3} & -L_{r, 3} P_{o r, 3}^{T}+\frac{1}{\epsilon}\left(I_{\left(n_{2}-l_{2}\right)}-\epsilon L_{r, 3} H_{r, 3}\right)
\end{array}\right]\left[\begin{array}{c}
\widehat{z}_{s, 3}(t) \\
\widehat{z}_{f \text { new } 2,3}(t)
\end{array}\right],
\end{aligned}
$$

$$
-\left(a_{44}^{* T}+\epsilon a_{24}^{* T} L_{r, 3}^{T}\right) H_{r, 3}^{T} .
$$

The matrices $P_{o r, 3}$ and $P_{o 2 r, 3}$ satisfy the algebraic Sylvester equation represented by

$$
\begin{aligned}
& \epsilon P_{o r, 3}\left(A_{s r, 3}^{T}-C_{s r, 3}^{T} K_{s r, 3}^{T}\right)-C_{f r, 3}^{T} K_{s r, 3}^{T}-A_{f r, 3}^{T} P_{o r, 3}=0 \Longrightarrow \\
& P_{o r, 3}=O(\epsilon) \\
& \epsilon P_{o 2 r, 3}\left(A_{s r, 3}-K_{s r, 3} C_{s r, 3}\right)-\epsilon K_{f 2 r, 3} C_{s r, 3} \\
& \quad-\left(A_{f r, 3}-K_{f 2 r, 3} C_{f \text { new } r, 3}\right) P_{o 2 r, 3}=0 \Longrightarrow \\
& P_{o 2 r, 3}^{0}=O(\epsilon) .
\end{aligned}
$$

The transformation is needed to relate both coordinates in which original observer (48) and parallel observer (68) are located; that is, where

$$
\begin{aligned}
T_{4 r, 3} & =\left[\begin{array}{cc}
I_{\left(n_{1}-l_{1}\right)} & P_{o r, 3}^{T}+H_{r, 3} \\
-L_{r, 3} & -L_{r, 3} P_{o r, 3}^{T}+\frac{1}{\epsilon}\left(I_{\left(n_{2}-l_{2}\right)}-\epsilon L_{r, 3} H_{r, 3}\right)
\end{array}\right], \\
T_{3 r, 3}^{-1} & =\left[\begin{array}{cc}
I_{\left(n_{1}-l_{1}\right)} & 0 \\
-P_{o 2 r, 3} & I_{\left(n_{2}-l_{2}\right)}
\end{array}\right] .
\end{aligned}
$$

Matrices $K_{s r, 3}^{*}$ and $(1 / \epsilon) K_{f 2 r, 3}^{*}$ are obtained from $T_{4 r, 3}^{-1} K_{z, 3}^{r}{ }^{r}$. The slow feedback gain $K_{s r, 3}$ can be obtained from the eigenvalue assignment problem; that is,

$$
\begin{aligned}
\lambda\left(A_{s r, 3}^{T}-C_{s r, 3}^{T} K_{s r, 3}^{T}\right) & =\lambda\left(A_{s r, 3}-K_{s r, 3} C_{s r, 3}\right) \\
& =\lambda_{s}^{\text {desired }}
\end{aligned}
$$

assuming that the following assumption is satisfied [14].

Assumption 7. The pair $\left(A_{s r, 3}, C_{s r, 3}\right)$ is observable.

The fast feedback gain $K_{f 2 r, 3}$ can be obtained from the eigenvalue assignment problem as

$$
\lambda\left(A_{f r, 3}-K_{f 2 r, 3} C_{f \text { new } r, 3}\right)=\lambda_{f}^{\text {desired }} .
$$

The following observability assumption is needed [14]. 


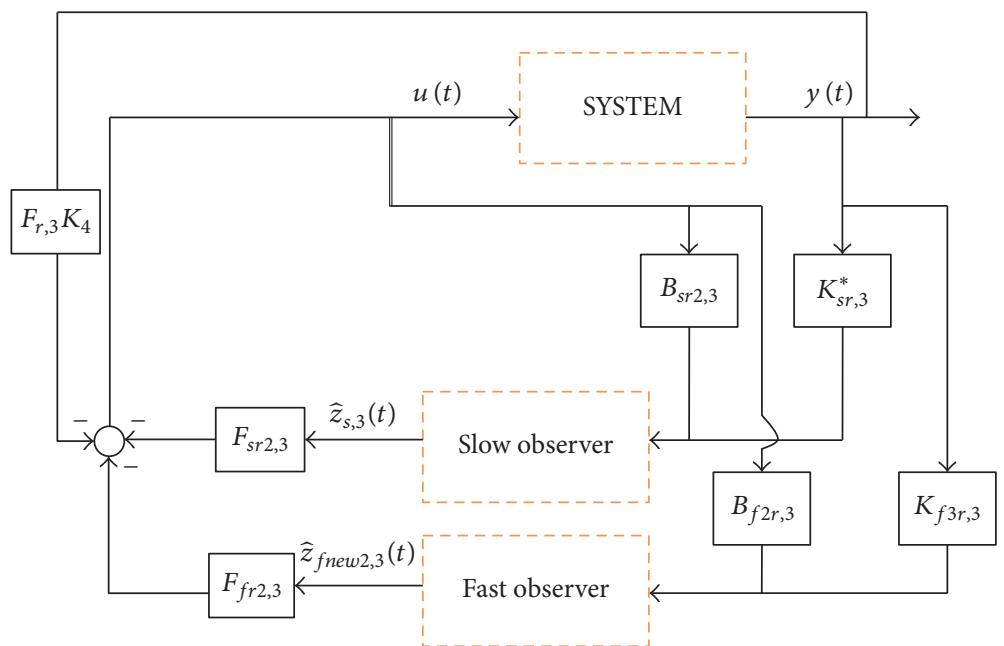

FiguRE 6: Case V: slow and fast observer-based controller design for a singularly perturbed linear system when some parts of the slow variables and some parts of the fast variables are measured.

The corresponding block diagram for the observer driven controller is presented in Figure 6.

\section{Numerical Example for Case I}

Consider a 4 th-order system with the system matrices $A, B$, and $C$ defined in [1]. The controllability matrix has full rank and therefore the pair $(A, B)$ is controllable. The results obtained using MATLAB are given below. We locate the feedback system slow eigenvalues at $\lambda_{c s}^{\text {desired }}=(-2,-3)$ and the feedback system fast eigenvalues at $\lambda_{c f}^{\text {desired }}=(-7,-8)$ and the reduced-order observer eigenvalues at $\lambda_{\text {robs }}^{\text {desired }}=$ $(-50,-60)$, given in the previous numerical example. Following the design procedure, the observer matrices $A_{z}, K_{z}, K_{11}$, $F_{1}$, and $F_{2}$ are given as

$$
\begin{aligned}
A_{z} & =\left[\begin{array}{cc}
-108.9999 & 0.2620 \\
-11034.3511 & -1.0000
\end{array}\right], \\
K_{11} & =\left[\begin{array}{ll}
0 & 314.5942 \\
0 & 31983.6265
\end{array}\right], \\
K_{z} & =\left[\begin{array}{cc}
0 & -259111.1037 \\
0 & -35033265.2948
\end{array}\right],
\end{aligned}
$$

and the feedback gains are obtained as

$$
\begin{aligned}
& F_{1}=\left[\begin{array}{ll}
92.9306 & 37.8637
\end{array}\right], \\
& F_{2}=\left[\begin{array}{ll}
2.4356 & 0.5349
\end{array}\right] .
\end{aligned}
$$

\section{Numerical Example for Case II}

Consider a 4 th-order system with the system matrices $A, B$, and $C$ defined in Section 5. We locate the feedback system slow eigenvalues at $\lambda_{c s}^{\text {desired }}=(-2,-3)$ and the feedback system fast eigenvalues at $\lambda_{c f}^{\text {desired }}=(-7,-8)$ and the reducedorder observer eigenvalues at $\lambda_{\text {robs }}^{\text {desired }}=(-50,-70)$, given in the previous numerical example. Following the design procedure, the observer matrices $A_{z}, K_{z}, K_{12}, F_{1}$, and $F_{2}$ are given as

$$
\begin{aligned}
A_{z} & =\left[\begin{array}{cc}
-49.9999 & 0.0000 \\
-0.0000 & -70.0000
\end{array}\right], \\
K_{12} & =\left[\begin{array}{ll}
11.0734 & 10.3042 \\
-6.4660 & 6.4112
\end{array}\right], \\
K_{z} & =\left[\begin{array}{cc}
-537.2325 & -556.1992 \\
400.7840 & -440.7330
\end{array}\right],
\end{aligned}
$$

and the feedback gains are

$$
\begin{aligned}
& F_{1}=\left[\begin{array}{ll}
92.9306 & 37.8637
\end{array}\right], \\
& F_{2}=\left[\begin{array}{ll}
2.4356 & 0.5349
\end{array}\right] .
\end{aligned}
$$

\section{Numerical Example for Case III}

Consider a 4th-order magnetic tape system from Section 5 . We locate the feedback system slow eigenvalues at $\lambda_{c s}^{\text {desired }}=$ $(-2,-3)$ and the feedback system fast eigenvalues at $\lambda_{c f}^{\text {desired }}=$ $(-7,-8)$ and the slow observer eigenvalues at $\lambda_{o s}^{\text {desired }}=-50$ and the fast observer eigenvalues at $\lambda_{o f}^{\text {desired }}=(-200,-300)$, given in the previous numerical example. Following the design procedure, the completely decoupled slow and fast observers in the $z_{s}-z_{f \text { new } 2}$ coordinates, driven by the system measurements and control inputs, are

$$
\begin{aligned}
\dot{\vec{z}}_{s}(t)= & {[-50.0000] \widehat{z}_{s}(t) } \\
& +[-2.323033338614758] u(t) \\
& +[-6196.4741] y(t) .
\end{aligned}
$$




$$
\begin{aligned}
& \dot{\vec{z}}_{f \text { new2 }}(t)=\left[\begin{array}{cc}
-1693.6295 & 113.2762 \\
-18376.0229 & 1193.6295
\end{array}\right] \widehat{z}_{f \text { new } 2}(t) \\
& +\left[\begin{array}{c}
-0.2499 \\
0.4666
\end{array}\right] u(t) \\
& +\left[\begin{array}{c}
-2859901.4120 \\
-33059040.2923
\end{array}\right] y(t) \\
& u(t)=-[1761.6414] \widehat{z}_{s}(t) \\
& -\left[\begin{array}{ll}
26.9824 & 5.1780
\end{array}\right] \widehat{z}_{\text {fnew2 }}(t)
\end{aligned}
$$

The slow and fast controller gains $F_{s r 2}$ and $F_{f r 2}$ are obtained as

$$
\begin{aligned}
& F_{s r 2}=[1761.6414], \\
& F_{f r 2}=\left[\begin{array}{ll}
26.9824 & 5.1780
\end{array}\right] .
\end{aligned}
$$

\section{Numerical Example for Case IV}

Consider a 4 th-order system with the system matrices $A, B$, and $C$ defined in Section 5 . We locate the feedback system slow eigenvalues at $\lambda_{c s}^{\text {desired }}=(-2,-3)$ and the feedback system fast eigenvalues at $\lambda_{c f}^{\text {desired }}=(-7,-8)$ and the slow observer eigenvalues at $\lambda_{o s}^{\text {desired }}=(-50,-60)$ and the fast observer eigenvalues at $\lambda_{\text {of }}^{\text {desired }}=-300$, given in the previous numerical example. Following the design procedure from Section 4, the completely decoupled slow and fast observer in the $z_{s}-z_{f \text { new } 2}$ coordinates, driven by the system measurements and control inputs, are

$$
\begin{aligned}
\dot{\vec{z}}_{s, 2}(t)= & {\left[\begin{array}{ll}
-0.0000 & -4127.7367 \\
0.7267 & -110.0000
\end{array}\right] \widehat{z}_{s, 2}(t) } \\
& +\left[\begin{array}{c}
4.7409 \\
-3.9951
\end{array}\right] u(t) \\
& +\left[\begin{array}{c}
113729.6251 \\
2281.0457
\end{array}\right] y(t) \\
\dot{\vec{z}}_{\text {fnew } 2,2}(t)= & -300.0000] \widehat{z}_{f \text { new } 2,2}(t) \\
& +[-779.5149] u(t) \\
& +[437703.1601] y(t) \\
u(t)= & -[-6530.324291 .6868] \widehat{z}_{s}(t) \\
& -[332.8512-10.9008] \widehat{z}_{f \text { new } 2}(t) .
\end{aligned}
$$

The slow and fast controller gains $F_{s r 2,2}$ and $F_{f r 2,2}$ are obtained as

$$
\begin{aligned}
& F_{s r 2,2}=\left[\begin{array}{ll}
-6530.3242 & 91.6868
\end{array}\right], \\
& F_{f r 2,2}=\left[\begin{array}{ll}
332.8512 & -10.9008
\end{array}\right]
\end{aligned}
$$

\section{Numerical Example for Case V}

Consider a 4 th-order system with the system matrices $A, B$, and $C$ defined in Section 5. We locate the feedback system slow eigenvalues at $\lambda_{c s}^{\text {desired }}=(-2,-3)$ and the feedback system fast eigenvalues at $\lambda_{c f}^{\text {desired }}=(-7,-8)$ and the slow observer eigenvalues at $\lambda_{o s}^{\text {desired }}=-50$ and the fast observer eigenvalues at $\lambda_{\text {of }}^{\text {desired }}=(-200,-300)$, given in the previous numerical example. Following the design procedure, the completely decoupled slow and fast observers in the $z_{s, 3^{-}}$ $z_{f \text { new } 2,3}$ coordinates, driven by the system measurements and control inputs, are

$$
\begin{aligned}
& \dot{\vec{z}}_{s, 3}(t)=[-50.0000] \widehat{z}_{s}(t) \\
& +[-2.323033338614758] u(t) \\
& +[-6196.4741] y(t) \\
& \dot{\vec{z}}_{f \text { new } 2,3}(t)=\left[\begin{array}{cc}
-1693.6295 & 113.2762 \\
-18376.0229 & 1193.6295
\end{array}\right] \widehat{z}_{f \text { new2 }}(t) \\
& +\left[\begin{array}{c}
-0.2499 \\
0.4666
\end{array}\right] u(t) \\
& +\left[\begin{array}{c}
-2859901.4120 \\
-33059040.2923
\end{array}\right] y(t) \\
& u(t)=-\left[\begin{array}{ll}
-6530.3242 & 91.6868
\end{array}\right] \widehat{z}_{s}(t) \\
& -\left[\begin{array}{ll}
332.8512 & -10.9008
\end{array}\right] \widehat{z}_{f \text { new2 }}(t) .
\end{aligned}
$$

The slow and fast controller gains $F_{s r 2,3}$ and $F_{f r 2,3}$ are obtained as

$$
\begin{aligned}
& F_{s r 2,3}=\left[\begin{array}{ll}
-6530.3242 & 91.6868
\end{array}\right], \\
& F_{f r 2,3}=\left[\begin{array}{ll}
332.8512 & -10.9008
\end{array}\right] .
\end{aligned}
$$

\section{Conclusion}

We have designed with high accuracy the reduced-order observer-based controllers for singularly perturbed linear systems. The numerical ill-conditioning of the original problem is removed. We have demonstrated that the full-order singularly perturbed system can be successfully controlled with the state feedback coming from the reduced-order observer-based controllers fully designed on the subsystem levels. The two-stage method is successfully implemented for both the observer and controller designs.

\section{Appendix}

It is well known that the rank condition after scalar multiplication is unchanged

$$
\operatorname{rank}(\alpha A)=\operatorname{rank}(A) .
$$


In Case (II), the pair $\left(A_{11},(1 / \epsilon) A_{21}\right)$ is observable, which implies

$$
\operatorname{rank}\left[\begin{array}{c}
\frac{1}{\epsilon} A_{21} \\
\frac{1}{\epsilon} A_{21} A_{11} \\
\frac{1}{\epsilon} A_{21} A_{11}^{2} \\
\vdots \\
\frac{1}{\epsilon} A_{21} A_{11}^{n-1}
\end{array}\right]=\operatorname{rank}\left[\begin{array}{c}
A_{21} \\
A_{21} A_{11} \\
A_{21} A_{11}^{2} \\
\vdots \\
A_{21} A_{11}^{n-1}
\end{array}\right] .
$$

\section{Conflicts of Interest}

The authors declare that there are no conflicts of interest regarding the publication of this paper.

\section{References}

[1] H. Yoo, New methods for design of full- and reduced-order observers and observer-based controllers for systems with slow and fast modes [Doctoral Dissertation], Rutgers University, 2017.

[2] R. G. Phillips, "A two-stage design of linear feedback controls," IEEE Transactions on Automatic Control, vol. 25, no. 6, pp. 12201223, 1980.

[3] L. R. Anderson, "Pole placement and order reduction in twotime-scale control systems through Riccati iteration," Applied Mathematical Modelling: Simulation and Computation for Engineering and Environmental Systems, vol. 3, no. 1, pp. 93-101, 1982.

[4] P. V. Kokotovic, H. K. Khalil, and J. O'Reilly, Singularly Perturbation Methods in Control: Analysis and Design, Academic Press, New York, NY, USA, 1986.

[5] Z. Gajic and M.-T. Lim, Optimal Control of Singularly Perturbed Linear Systems with Applications: High Accuracy Techniques, Marcel Dekker, New York, NY, USA, 2001.

[6] J. O'Reilly, "Full-order observers for a class of singularly perturbed linear time-varying systems," International Journal of Control, vol. 30, no. 5, pp. 745-756, 1979.

[7] J. O'Reilly, "Dynamical feedback control for a class of singularly perturbed linear systems using a full-order observer," International Journal of Control, vol. 31, no. 1, pp. 1-10, 1980.

[8] B. Porter, "Singular perturbation methods in the design of observers and stabilising feedback controllers for multivariable linear systems," IEEE Electronics Letters, vol. 10, no. 23, pp. 494495, 1974.

[9] C. Kuehn, Multiple Time Scale Dynamics, Springer, 2015.

[10] D. S. Naidu and A. J. Calise, "Singular perturbations and time scales in guidance and control of aerospace systems: a survey," Journal of Guidance, Control, and Dynamics, vol. 24, no. 6, pp. 1057-1078, 2001.

[11] M. T. Tran and M. E. Sawan, "Low-order observer for discretetime systems with slow and fast modes," International Journal of Systems Science, vol. 15, no. 12, pp. 1283-1288, 1984.

[12] K. R. Shouse and D. G. Taylor, "Reduced-order discretetime recursive observer for linear continuous-time singularly perturbed systems," in Proceedings of the American Control Conference, pp. 2128-2129, 1993.

[13] T. S. Raymond, S. Bahram, J. S. Clement, and H. Gene, Design of Feedback Control Systems, Harcourt Brace Jovanovich College Publishers, 1994.

[14] C. T. Chen, Linear System Theory and Design, Holt, Rinehart and Winston, New York, NY, USA, 1984. 


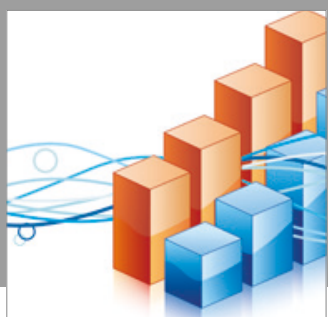

Advances in

Operations Research

vatersals

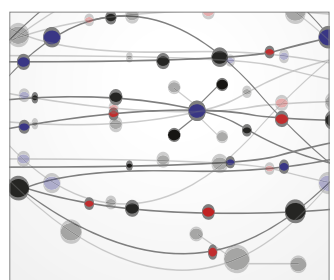

\section{The Scientific} World Journal
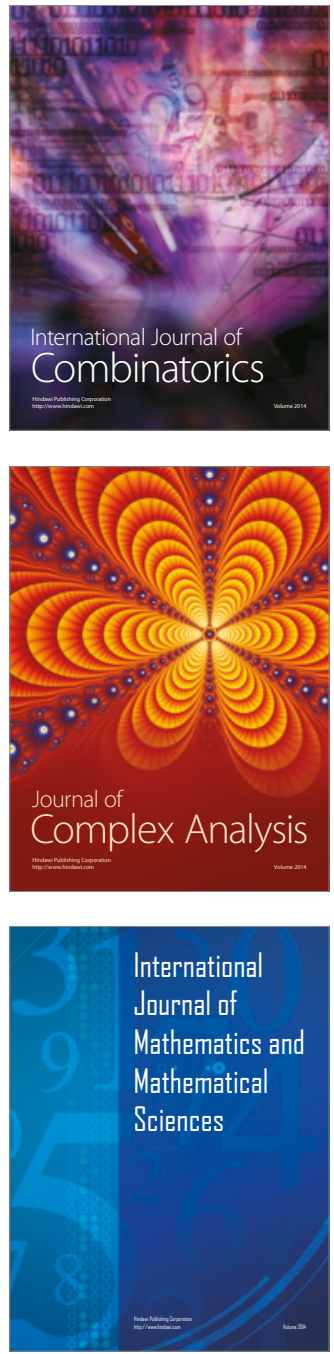
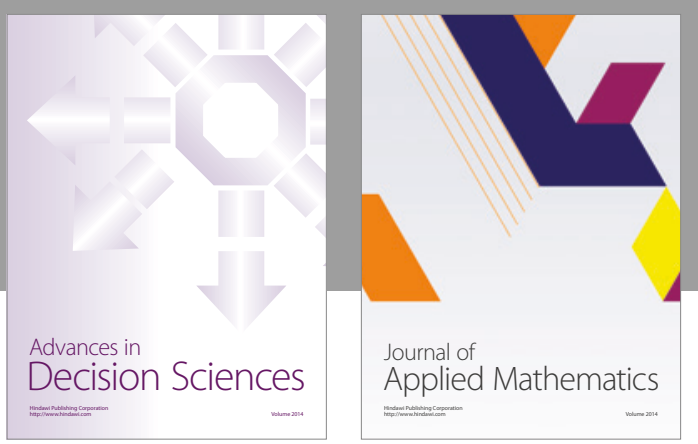

Algebra

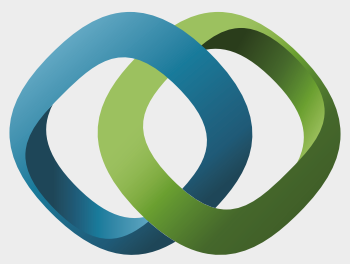

\section{Hindawi}

Submit your manuscripts at

https://www.hindawi.com
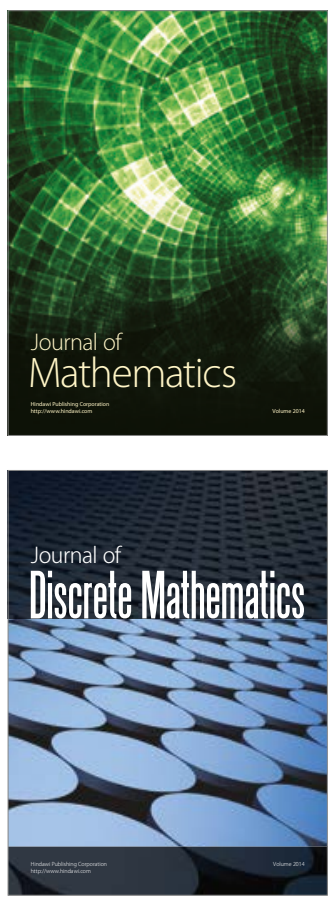

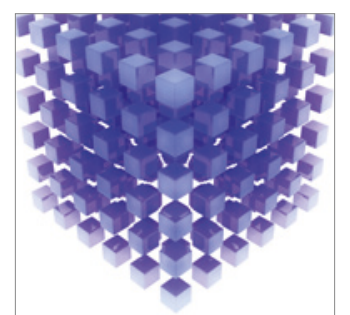

Mathematical Problems in Engineering
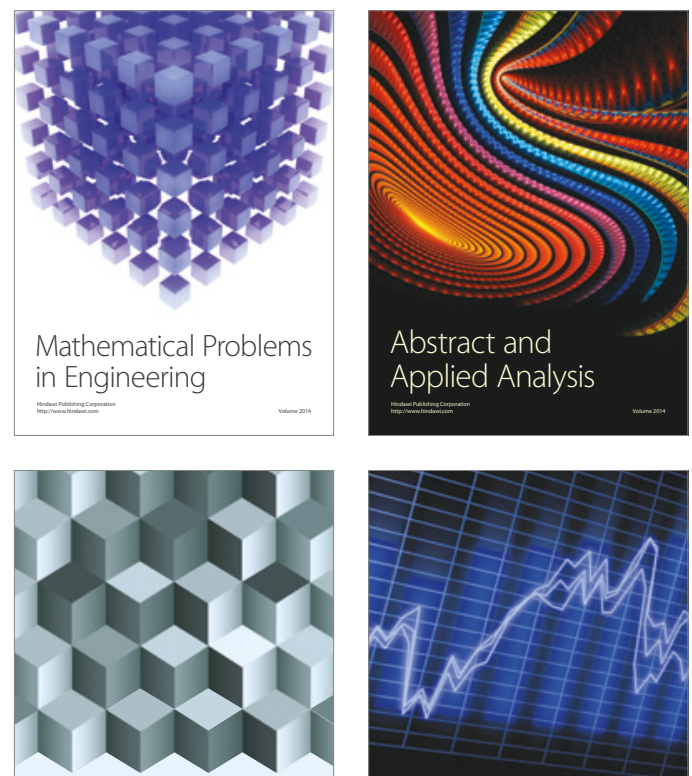

Journal of

Function Spaces

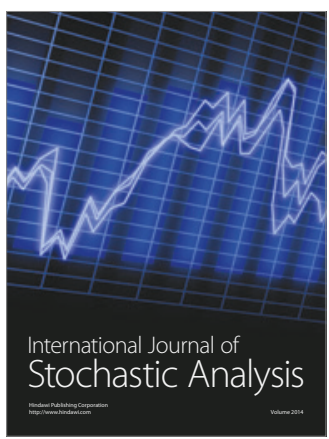

Probability and Statistics
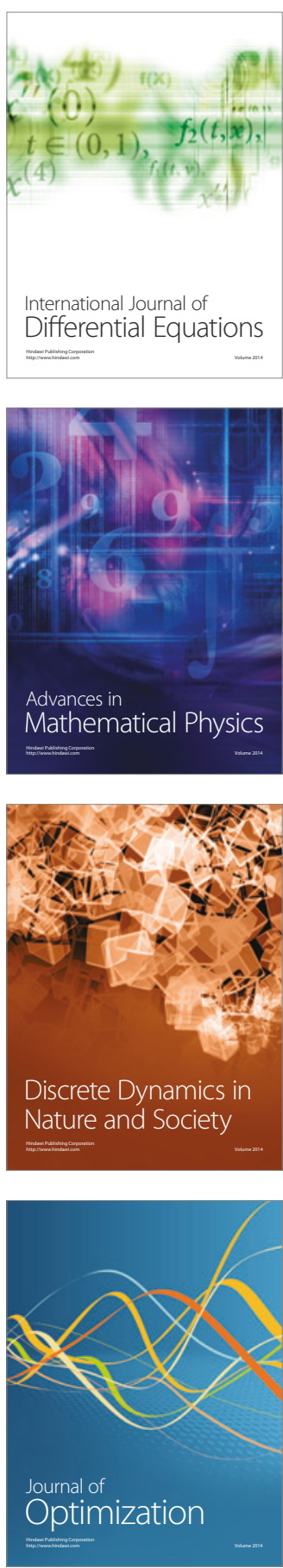